\title{
Factores asociados a la mortalidad de los pacientes atendidos por covid-19 en el servicio de urgencias
}

\author{
Dra. Karla María Delgado Guillen \\ karmari89@hotmail.com \\ https://orcid.org/0000-0003-0163-5895 \\ Hospital General Portoviejo \\ Dra. Maria Isabel Cedeño Ubillús \\ mauserrat_87@hotmail.com \\ https://orcid.org/0000-0001-9027-4627 \\ Hospital General IESS Portoviejo \\ Dra. Ana Isabel Zambrano Mendoza \\ jesusbajana1@gmail.com \\ https://orcid.org/0000-0002-3361-2674 \\ Hospital General Portoviejo \\ Dr. Wilson Xavier Morán Loor \\ xavman_2508@hotmail.com \\ https://orcid.org/ 0000-0001-9330-6129 \\ Hospital General Portoviejo \\ Dra. Arely Priscila Carranza Dominguez \\ prispkg@gmail.com \\ https://orcid.org/ 0000-0002-9790-5468 \\ Hospital General Portoviejo

\section{Dra. María Varynia Mendoza Santos \\ varynia ms@ hotmail.com https://orcid.org/0000-0002-6837-5162 Hospital General Portoviejo}

\section{RESUMEN}

La nueva enfermedad asociada al coronavirus, originada en China en 2019 y denominada enfermedad por coronavirus de 2019, es causada por un nuevo virus, llamado coronavirus de tipo 2 causante del síndrome respiratorio agudo grave, se caracteriza por su contagio directo, aparición de neumonía grave y peor evolución en adultos mayores o pacientes con comorbilidades como hipertensión, obesidad, diabetes o inmunosupresión, y por su rápida diseminación en el mundo, siendo la principal causa de muerte en este sigo XXI, la presente investigación fundamenta como Objetivo Describir los factores asociados a la mortalidad de los pacientes con COVID-19 que acuden al servicio de emergencias. Materiales y métodos. Se trata de un estudio observacional, retrospectivo, de casos y 
controles, analítico y transversal, se revisaron los expedientes digitales de 80 pacientes, en forma aleatoria con diagnóstico de Covid-19, que acudieron a la sala de urgencias del Hospital Básico San Andrés, como resultados El estudio revelo que del total de la muestra predominó el género masculino (77.3\%), también demuestra que la edad oscila con mayor frecuencia entre 40 a 60 años, factores asociados de forma independiente a la mortalidad intrahospitalaria

Palabras claves: pandemia; morbilidad; salud; covid-19. 


\title{
Factors associated with mortality in patients treated for covid-19 in the emergency department
}

\begin{abstract}
The new disease associated with the coronavirus, originated in China in 2019 and called the 2019 coronavirus disease, is caused by a new virus, called type 2 coronavirus that causes severe acute respiratory syndrome, is characterized by its direct contagion, the appearance of severe pneumonia and worse evolution in older adults or patients with comorbidities such as hypertension, obesity, diabetes or immunosuppression, and due to its rapid spread in the world, being the main cause of death in this 21 st century, the present research bases as Objective Describing the factors associated with the mortality of COVID-19 patients who come to the emergency service. Materials and methods. This is an observational, retrospective, case-control, analytical and cross-sectional study, the digital files of 80 patients were randomly reviewed with a diagnosis of Covid-19, who went to the emergency room of the San Andres Basic Hospital, as results The study revealed that of the total sample the male gender predominated (77.3\%), it also shows that age ranges more frequently between 40 to 60 years, factors independently associated with in-hospital mortality
\end{abstract}

Keywords: pandemic; morbidity; health; covid-19.

Artículo recibido: 02 Setiembre. 2021 Aceptado para publicación: 30 Setiembre. 2021

Correspondencia: karmari89@hotmail.com Conflictos de Interés: Ninguna que declarar 


\section{INTRODUCCIÓN}

La nueva enfermedad asociada al coronavirus, originada en China en 2019 y denominada enfermedad por coronavirus (COVID-19, por sus siglas en inglés), es causada por un nuevo virus, llamado coronavirus de tipo 2 causante del síndrome respiratorio agudo grave (SARS-CoV-2, por sus siglas en inglés). Se caracteriza por su contagio directo, aparición de neumonía grave y peor evolución en adultos mayores o pacientes con comorbilidades como hipertensión, obesidad, diabetes o inmunosupresión, y por su rápida diseminación en el mundo (Poutown Velavan \& Graners Meyer, 2020)' siendo considerada pandemia por la Organización Mundial de la Salud el 11 de marzo del 2020 (Organizacion Mundial de la Salud, 2020).

El 30 de enero de 2020, el Comité de Emergencias de la OMS declaró una emergencia sanitaria mundial en función de las crecientes tasas de notificación de casos en lugares chinos e internacionales. Las tasas de detección de casos en sus inicios eran cambiantes a diario y se podía rastrear casi en tiempo real en el sitio web proporcionado por la Universidad Johns Hopkins y otros foros. A mediados de febrero de 2020, China soporto la gran carga de morbilidad y mortalidad, mientras que la incidencia en otros países asiáticos, europeos y América del Norte seguía siendo baja hasta un determinado momento.

La pandemia por SARS-CoV-2 condiciona elevadas tasas de mortalidad en pacientes mayores hospitalizados o que acudían a las salas de emergencias. Actualmente, pocos estudios incluyen pacientes octogenarios y ninguno analiza el impacto del estado funcional sobre este resultado de salud (Figiredo Medeiros, 2021).Los estudios científicos demuestran que las medidas de distanciamiento social, son importantes para reducir la velocidad del crecimiento de la curva de incidencia y para prevenir un colapso en los sistemas de salud. Sin embargo, estas medidas tienen un gran impacto económico y social, lo que las hace excepcionales y temporales. Paradójicamente, cuanto más efectivas sean estas medidas, mayor será ( Gormed Hemida, 2020) el número de personas siguen siendo susceptibles al virus en las nuevas ondas epidémicas. Recientemente, países que ya tuvieron una reducción en el contagio, como Japón, han tenido que volver a adoptar medidas de distanciamiento social por un nuevo aumento del número de casos, poniendo en manifiesto el riesgo de las nuevas ondas epidémicas.

Cuando ocurre una Emergencia o Catástrofe, el Sistema de Salud en su conjunto, desde 
la propia Atención Primaria, los Servicios de Emergencias y Urgencias Hospitalarias, junto con los Hospitales en su conjunto, desempeñan un papel integral dentro del sistema de atención médica al proporcionar servicios médicos esenciales para atender a la sociedad.

Cualquier incidente que cause pérdida de infraestructura o aumento de pacientes, como un desastre natural, un acto terrorista, un accidente químico, biológico, radiológico, nuclear o explosivo, a menudo requiere una respuesta multijurisdictional y multifuncional que incluye un enorme esfuerzo de recuperación, que debe incluir la provisión de atención médica. Sin una apropiada planificación de la Emergencia, los sistemas de salud locales pueden verse colapsados fácilmente al intentar brindar atención durante un evento crítico. Los recursos son limitados, y ante un aumento en la demanda de servicios médicos, que puede verse acompañada de la interrupción de las comunicaciones y de las líneas de suministro, lo que supone una interrupción importante para la provisión adecuada de la atención médica (Sociedad Española de Medicina de Urgencia y Emergencia, 2020) El signo clínico inicial de la enfermedad COVID-19 relacionada con el SARS-CoV-2 que permitió la detección de casos fue la neumonía. Los informes más recientes también describen síntomas gastrointestinales e infecciones asintomáticas, especialmente entre los niños pequeños (Lvov \& Alkhovsky, 2020). Hasta ahora, las observaciones sugieren un período de incubación medio de cinco días y un período de incubación medio de 3 días (rango: 0-24 días). La proporción de personas infectadas por SARS-CoV-2 que permanecen asintomáticas durante el curso de la infección aún no se ha evaluado definitivamente.

En los pacientes sintomáticos, las manifestaciones clínicas de la enfermedad suelen comenzar en menos de una semana y consisten en fiebre, tos, congestión nasal, fatiga y otros signos de infecciones del tracto respiratorio superior. La infección puede progresar a una enfermedad grave con disnea y síntomas torácicos graves correspondientes a neumonía en aproximadamente el $75 \%$ de los pacientes al ingreso a emergencias ( Gormed Hemida, 2020). La neumonía ocurre principalmente en la segunda o tercera semana de una infección sintomática. Los signos prominentes de neumonía viral incluyen disminución de la saturación de oxígeno, desviaciones de los gases en sangre, cambios visibles a través de radiografías de tórax y otras técnicas de imagen, con anomalías en vidrio deslustrado, consolidación en parches, exudados alveolares y compromiso 
interlobulillar, lo que eventualmente indica deterioro. La linfopenia parece ser común y los marcadores inflamatorios (proteína $\mathrm{C}$ reactiva y citocinas proinflamatorias) están elevados.

La COVID-19 se asocia a manifestaciones psiquiátricas y neurológicas, entre las que se incluyen la ansiedad, la depresión, los trastornos del sueño, las cefaleas, los mareos, el deterioro del sentido del olfato o el gusto (Etienne, 2018), las mialgias, el delirio confusional o las encefalopatías, la excitación psicomotora, los accidentes cerebrovasculares, las lesiones cerebrales isquémicas hipóxicas, las convulsiones, el coma, las meningoencefalitis y el síndrome de Guillain Barré. Parece ser que es frecuente que las personas hospitalizadas por COVID-19 presenten ansiedad y depresión. En una cohorte de pacientes hospitalizados de Wuhan (China) más del 34\% de las personas cursaron con síntomas de ansiedad, y el 28\% con síntomas de depresión ( Yan, y otros, 2021).

Estudios internacionales han reportado características de los pacientes hospitalizados con COVID-19 asociados a mortalidad tales como la edad y comorbilidades como las enfermedades cardiovasculares, diabetes y obesidad ( Cummings, y otros, 2020) ( Grasselli, Greco, \& Zanella, 2020). En el país un estudio realizado en un hospital del Ministerio de Salud reporta una alta mortalidad respecto a otras series, y encuentran como hallazgo a la edad y la saturación de oxígeno al ingreso como principales factores predictores de mortalidad (Mejía, y otros, 2020)

La edad materna avanzada, un índice de masa corporal (IMC) elevado, ser de una etnia distinta a la blanca, referir enfermedades concomitantes preexistentes y presentar hipertensión crónica o diabetes preexistente son factores de riesgo para presentar un tipo grave de COVID-19. Aunque parece ser que las complicaciones relacionadas con la COVID-19 no aumentan en las mujeres que contraen la enfermedad en el tercer trimestre de la gestación en comparación con las que la contrajeron en uno de los primeros trimestres, ni en las multíparas en comparación con las primíparas, el tamaño de las muestras de las que se dispone para realizar para esas comparaciones es reducido. Apenas comienzan a entenderse las secuelas de la COVID-19 que se presentan a medio y largo plazo. 


\section{DESARROLLO}

La finalidad de este estudio fue describir las características de los pacientes adultos hospitalizados en el Hospital Básico San Andrés y determinar los factores asociados a mortalidad durante la hospitalización que permitan evaluar y proponer futuras estrategias para optimizar el manejo actual de los pacientes hospitalizados por COVID-19.

\section{Características al ingreso}

Durante el periodo de estudio ingresaron a la sala de emergencias un total de 80 adultos con el diagnóstico de COVID-19 confirmado, de los cuales 20 no cumplieron con los criterios de inclusión y fueron excluidos del estudio. De los pacientes con casos confirmados, 45 fueron diagnosticados mediante PCR y 15 por hisopado. La media de la edad fue de 40 años y 60 (70.5\%) dejando en evidencia que predomina el sexo masculino (Ver tabla 1).

Tabla1. Características de ingreso

\begin{tabular}{|c|c|c|c|c|c|c|}
\hline $\mathbf{N}^{\circ}$ de pacientes & Femenino & $\%$ & Masculino & \% & PCR & Hisopado \\
\hline 60 & 22 & 36,67 & 38 & 63,33 & 45 & 15 \\
\hline
\end{tabular}

Fuente: Elaboración propia

Los factores más frecuentes de mortalidad en los pacientes que ingresaron al área de emergencia fueron: hipertensión arterial (HTA) (60\%), obesidad (13,33\%) y diabetes mellitus (60\%) Insuficiencia renal crónica (15\%) En el caso particular de la diabetes, las infecciones virales son más difíciles de tratar, situación que se agrava en pacientes cuyos niveles de glicemia no están controlados, se conoce que en este grupo de pacientes el sistema inmune está comprometido. La HTA también es una preocupación, enfermedad que de por sí tiene complicaciones importantes para quien la padece, situación que se agrava en infección por SARS-CoV 2 (Ver tabla 2) 
Tabla 2. Comorbilidades

\begin{tabular}{|l|c|c|}
\hline \multicolumn{1}{|c|}{ Comorbilidades } & Total & \% \\
\hline Hipertensión arterial & 36 & 60 \\
\hline Obesidad & 8 & 13,33 \\
\hline Diabetes Mellitus & 36 & 60 \\
\hline Enfermedad renal crónica(III-IV-V) & 9 & 15 \\
\hline Cardiopatía Isquémica & 2 & 3,33 \\
\hline Cáncer & 3 & 5 \\
\hline Enfermedad cerebrovascular & - & 1,67 \\
\hline Hipotiroidismo & 1 & 1,67 \\
\hline VIH & 1 & 6,67 \\
\hline Enfermedad pulmonar intersticial difusa & 4 & \\
\hline Cirrosis hepática & - & 3,33 \\
\hline Enfermedad pulmonar obstructiva crónica & 2 & \\
\hline Antecedentes de Tuberculosis & & \\
\hline
\end{tabular}

Fuente: Elaboración propia

La mayor parte de los pacientes llegó al servicio de emergencia con clínica de mialgias, disnea, tos, artralgias, alteración olfatica. Independientemente del motivo de ingreso siendo Covid positivo, la mayoría de los pacientes arribó a la emergencia con sintomatología respiratoria, los síntomas al momento del ingreso más relevantes fueron disnea (81.67\%), tos (86.67\%), hipertermia (80\%), malestar general, alteración olfatoria, disgeusia y artralgias coinciden con un (88.33\%), congestión nasal (61.67) (Ver tabla 3)

Tabla 3. Síntomas de Ingreso

\begin{tabular}{|l|c|c|}
\hline \multicolumn{1}{|c|}{ Síntomas } & Total & \% \\
\hline Disnea & 49 & 81,67 \\
\hline Tos & 52 & 86,67 \\
\hline Hipertermia & 48 & 80 \\
\hline Malestar general & 53 & 88,33 \\
\hline Odinofagia & 28 & 46,67 \\
\hline Cefalea & 45 & 75 \\
\hline Disgeusia & 53 & 88,33 \\
\hline Congestión nasal & 37 & 61,67 \\
\hline Alteración olfatoria & 53 & 88,33 \\
\hline Artralgias & 53 & 88,33 \\
\hline Asintomáticos & 7 & 11,67 \\
\hline
\end{tabular}

Fuente: elaboración propia.

Es importante lo que este estudio demuestra que indiferentemente de cualquiera que sea el diagnostico empleado de forma tardía, las complicaciones o comorbilidades, contribuyen a que estos pacientes con diagnósticos de Covid, se complique de tal manera como lo indica la tabla. De un total de pacientes que ingresaron a la Unidad de Cuidados 
Intensivos, 13 de 16 fallecieron, mientras que de aquellos que necesitaron oxígeno y requerimiento de UCI, solo 2 fallecieron.

Tabla 4. Definición de los casos y Diagnósticos

\begin{tabular}{|l|c|c|c|}
\hline Características & Total & Sobreviviente & Fallecidos \\
\hline Diagnóstico Clínico & 26 & 18 & 8 \\
\hline Diagnostico radiológico & 12 & 8 & 4 \\
\hline Prueba Molecular positivo & 3 & - & 3 \\
\hline Caso confirmado & 18 & 15 & 3 \\
\hline Tiempos de Hospitalización & & & \\
\hline Duración de síntomas previo al ingreso, días & 6 & - & - \\
\hline Estancia hospitalaria, días & 15 & - & - \\
\hline Desenlaces & & & 1 \\
\hline Requerimiento de altos flujos (FIO2 $\geq)$ & 30 & 29 & 1 \\
\hline Requerimiento de UCI & 14 & 13 & 13 \\
\hline Ingreso a UCI & 16 & 3 & \\
\hline
\end{tabular}

Fuente: Elaboración propia

La afectación clínica en los pacientes Covid-19 positivos, es evidente que existe una alteración sistemática de todos los sistemas y aparatos en el durante y después del alta médica. Las implicaciones funcionales de esta peculiar patogénesis desembocan el Síndrome de Distres Respiratorio Agudo (SDRA) en la COVID-19 incluye un empeoramiento progresivo de los desequilibrios de ventilación/perfusión y una pérdida de reflejos de vasoconstricción hipóxicas, con un componente marcado de trombosis pulmonar microvascular, creando a su paso un desequilibrio sistémico. 
Tabla 5. Afectación clínica secular y consecuencias del Coronavirus más frecuentes

\begin{tabular}{|c|c|}
\hline Tejido o sistema & Síntomas seculares asociados a la Covid-19 \\
\hline Vía aérea respiratoria superior & - Grado residual de anosmia y/o ageusia Tos \\
\hline Vía aérea respiratoria inferior & $\begin{array}{l}\text { - Disnea de esfuerzo } \\
\text { - Tos } \\
\text { - Secreción mucosa } \\
\text { - Dificultad para inspiración profunda } \\
\text { - Dolor torácico }\end{array}$ \\
\hline Muscular & $\begin{array}{l}\text { - Debilidad generalizada } \\
\text { - Dolores erráticos }\end{array}$ \\
\hline Neurocognitivo & $\begin{array}{l}\text { - Falta de atención } \\
\text { - Pérdida de memoria } \\
\text { - Mala calidad del sueño } \\
\text { - Insomnio }\end{array}$ \\
\hline Psicologico & $\begin{array}{l}\text { - Ansiedad } \\
\text { - Depresión }\end{array}$ \\
\hline Digestivo & $\begin{array}{l}\text { - Atragantamiento } \\
\text { - Cambio en el ritmo de posicional } \\
\text { - Sensación de plenitud }\end{array}$ \\
\hline Otros & - Pérdida de peso \\
\hline
\end{tabular}

Fuente: Elaboración propia

\section{DISCUSIÓN}

La pandemia por SARS CoV-2 está asociada con una alta morbi-mortalidad. La evidencia científica demostraría, en los pacientes con COVID-19, que las comorbilidades diabetes mellitus, hipertensión arterial(HTA), enfermedad pulmonar obstructiva crónica (EPOC) y las enfermedades cardio vasculares son las más prevalentes en asociación a la muerte del paciente. En el caso particular de la diabetes y la HTA son considerados factores de riesgo para un resultado desfavorable, sin embargo, aun la evidencia científica es Contradictorio ( Navarrete-Mejía, Lizaraso-Soto, Velasco Guerrero, \& Loro Chero, 2020) Respecto a las comorbilidades encontradas, la hipertensión arterial es la comorbilidad más frecuente seguida por diabetes y obesidad ( Vences, 2020). Sin embargo, el porcentaje de obesidad descrito en nuestro estudio es bajo y no se encontró asociado con mayor mortalidad, hallazgo descrito en otros estudios ( Valenzuela Casquino, Espinoza Venero, \& Quispe Galvez, 2020).

En un estudio observacional que se hizo en una serie de casos en Francia se determinó que el $65 \%$ de los enfermos de COVID-19 que fueron ingresados en unidades de cuidados 
intensivos presentó signos de confusión (o delirio confusional) y el 69\% refirió excitación psicomotora (Sociedad Española de Medicina Interna, 2020). Especialmente el delirio confusional se ha asociado a un aumento del riesgo de muerte en el marco de la COVID19. Además, la COVID-19 se ha asociado a accidentes cerebrovasculares (incluidos los tipos isquémico y hemorrágico)

De 655 paciente que presentaban COVID-19 confirmada mediante pruebas de laboratorio y en los que se disponía de información completa sobre los antecedentes personales patológicos, en el $23 \%$ del total existía alguna enfermedad preexistente; los procesos patológicos que se registraron con mayor frecuencia fueron la obesidad, las neumopatías crónicas (entre ellas el asma), las enfermedades cardiovasculares y distintos tipos de inmunodepresión.

\section{CONCLUSIONES}

- La diabetes y la hipertensión arterial están entre las comorbilidades que mayor riesgo implican, es una presentación clínica grave en pacientes con la COVID-19, los hallazgos son de importancia para el adecuado abordaje terapéutico de los pacientes afectados y para el desarrollo de estrategias de salud, en materia de prevención de complicaciones y tratamiento oportuno en el contexto de la pandemia.

- Nuestro estudio también muestra una marcada prevalencia de obesidad en los pacientes que tuvieron desenlace fatal. Estos hallazgos ya han sido descritos previamente mostrando que la obesidad es un rol del tejido adiposo y la expresión de enzimas supone un factor de riesgo para requerimiento de ventilación mecánica en pacientes hospitalizados con COVID-19.

- Los pacientes con Insuficiencia Cardiaca hospitalizados por COVID-19 tienen una alta tasa de mortalidad intrahospitalaria. Algunas pruebas clínicas y de laboratorio sencillas pueden ayudar a identificar a los pacientes con peor pronóstico.

- La patogenia de esta enfermedad, mejor conocida en la actualidad, explica las implicaciones en los casos críticos y graves con elevada mortalidad, sobre todo la población mayor de 60 años y patologías crónicas asociadas, aunque los de menor edad con las patologías referidas corren también el riesgo. El SARS-CoV-2 con compromiso pulmonar serio, complicaciones evolutivas y elevada mortalidad no es explicable solo por la inflamación del tejido pulmonar de una simple neumonía viral. 
Intervienen otros factores de mucha mayor gravedad: un proceso inflamatorio sistémico, con afectación multiorgánica y trastornos graves de la coagulación

\section{BIBLIOGRAFÍA}

Aragón, E., \& Zapata, G. (01 de 2016). Evaluación de la calidad de vida en pacientes con enfermedad renal crónica en tratamiento sustitutivo. Cambios, 16. doi:https://doi.org/10.36015/cambios.v15.n1.2016.179

Bianchini Matamoros, M. (2019). El Síndrome del Burnout en personal profesional de la salud. Scielo, 14. Recuperado el 08 de 2021, de https://www.scielo.sa.cr/scielo.php?script=sci_arttext\&pid=S140900151997000200017

Bravo Bonoso, D. G., Álava Castro, W. F., \& Álava Bravo, E. I. (2021). Docentes universitarios y su desempeño laboral. Revista Científica de la Universidad de las Ciencias Informáticas, 9. Recuperado el 08 de 06 de 2021, de https://publicaciones.uci.cu/index.php/serie/article/view/764/643

Cummings , M., Baldwin , M., Abrams, D., Jacobson, S., Meyer , B., Balough , E., . . Rabbani , L. (05 de 2020). Epidemiología, curso clínico y resultados de adultos críticamente enfermos con COVID-19 en la ciudad de Nueva York: un estudio de cohorte prospectivo. PubMed, 9. doi:10.1016 / S0140-6736 (20) 31189-2

Gormed Hemida, M. (2020). Las técnicas de diagnóstico de coronavirus de próxima generación con especial énfasis en el SARS-CoV-2. PudMed, 8. doi:10.1002 / jmv.26926

Grasselli, G., Greco, M., \& Zanella, A. (2020). Factores de riesgo asociados con la mortalidad en pacientes con COVID-19 en unidades de cuidados intensivos en Lombardía, Italia. Jama Network, 12. doi:10.1001 / jamainternmed.2020.3539

Hassard, J., \& Cox, T. (02 de 2021). Los riesgos psicosociales y el estrés en el trabajo. Recuperado el 20 de 08 de 2021, de https://osha.europa.eu/es/themes/psychosocial-risks-and-stress

Navarrete-Mejía, P. J., Lizaraso-Soto, F. A., Velasco Guerrero, J. C., \& Loro Chero, L. M. (2020). Diabetes mellitus e hipertensión arterial como factor de riesgo de mortalidad en pacientes con Covid-19. Revista Cuerpo Medico Hospital Nacional Almanzor Aguinaga Asenjo, 12. Recuperado el 04 de 09 de 2021, de oi.org/10.35434/rcmhnaaa.2020.134.766DOI361Rev. cuerpo méd. HNAAA 
13(4) 2020

Valenzuela Casquino, K., Espinoza Venero, A., \& Quispe Galvez, J. C. (2020). Mortalidad y factores pronósticos en pacientes hospitalizados por COVID-19 en la Unidad de Cuidados Intermedios de un hospital público de Lima, Perú. Horiz Med, 06. Recuperado el 04 de 09 de 2021, de http://www.scielo.org.pe/pdf/hm/v21n1/1727-558X-hm-21-01-e1370.pdf

Vences, M. A. (2020). Factores asociados a mortalidad en pacientes hospitalizados con COVID-19: cohorte prospectiva en un hospital de referencia nacional de Perú. Revista Medic, 17. doi:Doi 10.5867 / medwave.2021.06.8231

Yan, Y., Woo In Shin, Xin Pang, Y., Yang Meng, Jianchen Lai, Chong You , ... Heng Pang, C. (2021). Los primeros 75 días del brote del nuevo coronavirus (SARSCoV-2): avances recientes, prevención y tratamiento. International Journal of Environmental Research and Public Health, 17. Recuperado el 13 de 08 de 2021, de https://www.ncbi.nlm.nih.gov/pmc/articles/PMC7177691/

Andrés, A. G. (2020). Recuperado el 11 de 02 de 2021, de Repositorio Digital UEB: http://dspace.ueb.edu.ec/handle/123456789/3677?mode=full

Arroyo Santander, O. A. (2017). Riesgos psicosociales en el personal de enfermería que labora en el Hospital San Luis de Otavalo, 2016. Universidad Tecnica del Norte, Ibarra. Recuperado el 20 de 08 de 2021, de http://repositorio.utn.edu.ec/bitstream/123456789/6820/1/06\%20ENF\%20823\% 20TRABAJO\%20DE\%20GRADO.pdf

Bermeo Minchala, M. L., \& Jimenez Yora, J. V. (2014). Evaluacion de la calidad de vida de los pacientes con Insuficicencia Renal Cronica sometidos a hemodialisis. Azoguez. Recuperado el 04 de 06 de 2021, de http://dspace.ucuenca.edu.ec/bitstream/123456789/21563/1/Tesis\%20Pregrado.p df

Carlos, D. (2020). Recuperado el enero de 2021, de pichincha comunicaciones : pichinchacomunicaciones.com.ec/lapandemia-del-coronavirus-afecta-a-la-saludmental-de-millones-de-personas/.

Churata Salon, F. (2019). FACTORES PSICOSOCIALES Y MEDIDAS DE BIOSEGURIDAD DURANTE DESEMPEÑO LABORAL DE MÉDICOS RESIDENTE HOSPITAL GUILLERMO ALMENARA IRIGOYEN 2019. 
Universidad de San Martin de Porres, Peru. Recuperado el 08 de 2021, de https://repositorio.usmp.edu.pe/bitstream/handle/20.500.12727/6541/churata_sf. pdf? sequence $=3 \&$ is Allowed $=\mathrm{y}$

Coello, C. (Mayo de 2020). Trabajadores de la salud ya presentan síndrome del quemado e inestabilidad emocional. Edicion Medica.

Etienne, C. F. (octubre de 2018). Salud mental como componente de la salud universal. Revista Panamericana de Salud Pública.

Figiredo Medeiros, A. (2021). Factores asociados a la incidencia y la mortalidad por COVID-19 en las comunidades autónomas. Gaceta Sanitaria, 445'452. $\begin{array}{llllll}\text { Recuperado el de } & 01 \text { de }\end{array}$ https://www.sciencedirect.com/science/article/abs/pii/S001425652030179X

Herodes, E., \& Barbosa, J. (noviembre de 2020). Uno de cada cinco trabajadores de la salud experimenta síntomas de depresión. edicion medica.

Loaiza, Y. (2019). Recuperado el enero de 2021, de Revista GK: https://gk.city/2019/09/02/depresion-ansiedad-salud-mental/

Lvov , D., \& Alkhovsky, S. (2020). Fuente de la pandemia COVID-19: ecología y genética de coronavirus (Betacoronavirus: Coronaviridae) SARS-CoV, SARSCoV-2 (subgénero Sarbecovirus) y MERS-CoV (subgénero Merbecovirus). PubMed, 9. Recuperado el 31 de 08 de 2021, de https://pubmed.ncbi.nlm.nih.gov/32515561/

Maria, M. P. (Julio de 2018). Burnout y factores de riesgo psicosocial en el personal de un hospital de larga estancia. Scielo, 34(11). Recuperado el enero de 2021, de Revista Scielo: http://scielo.isciii.es/scielo.php?script=sci_arttext\&pid=S0465546X2011000500002

Mejía, F., Medina , C., Cornejo, E., Morello, E., Vasquez, S., Alave, J., \& Malaga , G. (2020). Características clínicas y factores asociados a mortalidad en pacientes adultos hospitalizados por COVID-19 en un hospital público de Lima, Perú. Scielo, 23. Recuperado el 02 de 09 de 2021, de 10.1590/SciELOPreprints.858

OMS. (2018). Recuperado el 31 de ENERO de 2021, de Organizacion Mundial de la Salud: $\quad$ https://www.who.int/es/news-room/fact-sheets/detail/mental-healthstrengthening-our-response

Organizacion Mundial de la Salud. (2020). COVID-19; Coronavirus; Ética en 
Investigación; Bioética. Organizacion Mundial de la Salud, Estados Unidos. Recuperado el 13 de 03 de 2021, de https://iris.paho.org/handle/10665.2/52142 Organizacion Mundial de la Salud. (2020). OMS. Recuperado el ENERO de 2021, de https://www.who.int/es/news/item/05-10-2020-covid-19-disrupting-mentalhealth-services-in-most-countries-who-survey

Poutown Velavan, T., \& Graners Meyer, C. (2020). La epidemia de COVID-19. TMIH Journal, 9. Recuperado el 1 de 08 de 2021, de https://onlinelibrary.wiley.com/doi/10.1111/tmi.13383

Puente, A. (Marzo de 2020). Impacto de la epidemia del Coronavirus (COVID-19) en la salud mental del personal de salud y en la población general de China. Scielo .

Sociedad Española de Medicina de Urgencia y Emergencia. (2020). Planes de Contingencia Pandemia COvid-19. España. Recuperado el 31 de 08 de 2021, de https://www.semes.org/wp-content/uploads/2020/07/MANUAL-DE-

ACREDITACION-ESCALADA-DESESCALADA-DE-HOSPITALESPANDEMIA-COVID19-1.pdf

Sociedad Española de Medicina Interna. (2020). XXVII CONGRESO NACIONAL DE LA SOCIEDAD ESPANOLA DE MEDICINA INTERNA (SEMI). Salamanca. $\begin{array}{llllll}\text { Recuperado el de } & 04 \text { de }\end{array}$ https://www.fesemi.org/sites/default/files/documentos/congresos/librocomunicaciones-1.pdf

Soraya, L. O. (2017). Factores de riesgo psicosocial y su relacion con la salud mental en los enfermeros. Tesis para obtencion de maestría, Universidad Católica de Santa María, SAalud ocupacional y medio abiente, Peru.

Tesillo, S. Y., \& Martínez, A. O. (junio de 2018). Burnout en profesionales de la salud. Psicologia y Salud, 28(1: 63-72).

Trabajo, O. I., \& Salud, O. M. (1988). Factores psicosociales en el trabajo: naturaleza, incidencia y prevención. Informe del Comité mixto OIT/OMS sobre medicina del trabajo. Geneva: Organización Internacional del Trabajo/Organización Mundial de la Salud. 